\title{
Rheumatic Fever and Rheumatic Heart Diseases in Bangladesh: Challenges and Remedies
}

\author{
Mohammad Arifur Rahman ${ }^{1}$, Afzalur Rahman ${ }^{1}$, Syed Nasir Uddin ${ }^{1}$, AKM Monwarul Islam ${ }^{1}$, Tariq \\ Ahmed Chowdhury ${ }^{1}$, Farhana Ahmed ${ }^{1}$, Sharadindu Shekhar Ray ${ }^{1}$, Delwar Hossain ${ }^{1}$, Laila Farzana ${ }^{2}$ \\ ${ }^{1}$ Department of Cardiology, NICVD, Dhaka, ${ }^{2}$ Family Red Crescent Medical College and Hospital
}

\begin{abstract}
Key words:

In the $21^{\text {st }}$ century, Rheumatic fever (RF) and Rheumatic heart disease (RHD) are neglected diseases of Rheumatic

fever, Rheumatic

Heart Disease. marginalized communities. Globally, RHD remains the most-common cardiovascular disease in young people aged $<25$ years. Although RF and RHD have been almost eradicated in areas with established economies, migration from low-income to high-income settings might be responsible for a new burden of RHD in high-income countries. Globally, the prevalence of rheumatic fever (RF) and rheumatic heart disease (RHD) has declined sharply but, in developing countries, RF is still a-- leading cause of heart disease and, consequently, death in children and young adults. In 2005, it was estimated that over 2.4 million children aged 5-14 years were having RHD globally and 79\% of all these cases were from lessdeveloped countries.
\end{abstract}

(Cardiovasc. j. 2018; 10(2): 206-211)

\section{Introduction:}

Globally, the prevalence of rheumatic fever (RF) and rheumatic heart disease (RHD) has declined sharply but, in developing countries, $\mathrm{RF}$ is still a-- leading cause of heart disease and, consequently, death in children and young adults. ${ }^{1}$ In 2005, it was estimated that over 2.4 million children aged 5-14 years were having RHD globally, and $79 \%$ of all these cases were from less-developed countries, such as Bangladesh. ${ }^{2}$ The prevalence of RF defined by the revised Jones criteria among children aged 5-15 years in rural Bangladesh was 1.2. ${ }^{3}$ These are conservative estimates, especially if echocardiographic screening is used; the actual figures are likely to be substantially higher. ${ }^{4}$ Zaman et al. opined that protein energy malnutrition is likely to be associated with RF. ${ }^{5}$

Excluding the developed economies, the global burden of RHD in the 5-14 year old children has been estimated to be $0.8-5.7 / 1000$ with a median of $1.3 / 1000,{ }^{6}$ while the overall incidence of acute $\mathrm{RF}$ varies from 5 to $51 / 100,000$ population with a mean of $19 / 100,000 .{ }^{7}$ In a recent systematic review, the greatest burden of RF and RHD was found in sub-Saharan Africa, the lowest in North America, the highest mortality rates in the indigenous populations of Australia (23.8/ 100,000), and among the countries with World Health Organization (WHO) vital registration data, the highest mortality was found in Mauritius (4.32/ 100,000). ${ }^{8}$ The exact incidence and prevalence of RF and RHD in Bangladesh are not known. In the $2^{\text {nd }}$ half of last century, $\mathrm{RF}$ and RHD constituted a significant proportion of admissions in general hospitals, and a lion's share of cardiovascular admissions. ${ }^{9}$ Probably the community prevalence of RF and RHD was first reported in 1976, which was 7.5/1000 in general population. ${ }^{10}$

\section{Discussion:}

Rheumatic fever is prevalent in many of the developing countries of the Indian subcontinent, the Arab countries of the Middle East, and the urban metropolises of Central and South America and Africa. ${ }^{11}$ In developed countries, it has shown a decreasing trend for more than half a century and become almost absent nowadays. However, the trend in the developing countries remains almost unknown. It is extremely difficult to ascertain the exact trend in a developing country like Bangladesh. ${ }^{12}$

A community-based study was done on 5923 rural Bangladeshi children aged 5-15 years to

Address of Correspondence: Dr.Mohammad Arifur Rahman, Department of Cardiology, National Institute of Cardiovascular Diseases, Dhaka, Bangladesh. Email: drarif79@yahoo.com 
determine the prevalence of rheumatic fever (RF) and rheumatic heart disease (RHD). The prevalence was found to be 1.2 (95\% confidence interval 0.3-2.1) per 1000 for RF defined by revised Jones criteria and $1.3(0.4-2.2)$ per 1000 for Doppler echocardiography-confirmed RHD. ${ }^{13}$

Challenges and Remedies: Bangladesh Dealing with Controlling Rheumatic Fever and Rheumatic Heart Disease:

Bangladesh is always vigilant to curb ARF and RHD with their sequels. Both Government and non government agencies working together in this field. There is a dedicated centre located at Dhaka known as National Center for Control of Rheumatic Fever and Heart Diseases (NCCRF\&HD) to control RF and RHD. There is another centre known as National Institute of Cardiovascular Diseases (NICVD) for treatment of RF, RHD and to manage their complications specially Percutaneous transluminal mitral commissurotomy (PTMC), Closed mitral commissurotomy (CMC), Open mitral commissurotomy (OMC) and Valve replacement etc.

Following are the stepwise approaches Bangladesh adopted to face the challenges of RF and RHD.

1. Understanding of disease pathogenesis to improve management

A better understanding of ARF/RHD pathogenesis is critical to developing preventive and therapeutic interventions. Current approaches to diagnosis and treatment of ARF have their foundations in the mid- $20^{\text {th }}$ Century ${ }^{14}$ The Jones Criteria have been revised and updated several times, and some countries have adapted them for their own circumstances, ${ }^{15}$ but there continues to be cases of under- and over diagnosis, often with tragic consequences. ${ }^{16}$

\section{A. Immunology of the disease}

We need prospective, active recruitment of ARF cases with a view to using recent technologies to unravel the mysteries of the pathogenesis, particularly the immune response, of ARF. Using standardized data collection forms, each case would undergo thorough immune-phenotypic profiling, drawing on the latest understanding of other autoimmune diseases as well as the latest technologies.

\section{B. Genetics of this condition}

There is an inherited susceptibility to ARF, ${ }^{17}$ but the basis for this susceptibility has yet to be clarified. ${ }^{18}$ We have never sought genetic markers for ARF/RHD using modern techniques. We need to use these techniques to determine if there are areas of the human genome with polymorphisms highly associated with $\mathrm{ARF} /$ RHD, with a view to sequencing and further studying those areas of the genome to identify the nature of host susceptibility.

\section{Early Identification to Increase Chance of Success}

It is the unfortunate reality that most new patients entered onto $\mathrm{ARF} / \mathrm{RHD}$ registers have already developed significant rheumatic valvular lesions. A major challenge, therefore, is to identify people with RHD early in the natural history of their illness, during a phase that often lasts many years when most cases are asymptomatic. The only way to identify asymptomatic cases is to conduct screening. We and others are increasingly demonstrating that echocardiographic screening is preferable to auscultation, ${ }^{19}$ but many unanswered questions remain before this can be routinely advocated.

A. Standardization by echocardiographic screening for rheumatic heart disease

The aim is to share screening echocardiograms through a web-based interface, to compare interpretations by cardiologists and others around the world, with a view to making echo reading as objective as possible. The aim is for all echo readers to undergo training using a standard set of echocardiograms and agreed diagnostic criteria, with measurement of interand intra-observer variability before proceeding to read new screening echocardiograms.

B. Evidence-based diagnostic criteria for rheumatic heart disease

In 2005, a joint NIH-WHO working group developed standardized surveillance protocols for GAS diseases, which included a diagnostic definition of RHD on echocardiogram. This was a consensus definition based on opinion of an expert group, because of the absence of data. ${ }^{20}$ The global collaboration needs to consolidate these data to develop evidence-based diagnostic criteria for RHD on echo. 
C. Determining the significance of subclinical carditis

There is concern that the extremely high rates of subclinical RHD being found in some studiesoften ten times more that found by relying on the presence of a significant cardiac murmurhas not been proven to represent true RHD, Conversely, this may indeed represent a massive undetected burden of RHD, but we have no confirmation that these cases are truly part of the spectrum of clinical RHD, that they may potentially progress to clinical disease, and that they would benefit from secondary prophylaxis.

D. Determining the cost-effectiveness of screening, and making it justifiable

All of this information needs to be compiled in an objective case for screening, complete with economic analysis. If screening is warranted, the challenge then is to make it practical and scalable in developing countries. In all countries, routine screening of school-aged children is not feasible if it relies on highly trained echocardiography technicians using expensive portable machines. The aim would be to generate guidelines for screening accompanied by training curricula for inexpert screening echocardiographers.

\section{Search for an Effective Approach to Primary Prevention}

\section{A. Vaccine for rheumatic fever}

In March 2010, the new Hilleman Institute (collaboration between the Wellcome Trust and Merck, having established an institute in India charged with developing vaccines for lessdeveloped countries) convened a meeting to determine if their first priority vaccine would be for group A streptococcus (GAS). The meeting was a turning point for GAS disease control. It quickly became clear that GAS vaccines would not be chosen for several reasons reason. The current understanding of the immunopathogenesis of GAS diseases, particularly $\mathrm{RF} /$ RHD, is crude, and investigators have not taken advantage of the latest technologies.

The role of primary prophylaxis of streptococcal sore throat

There is a disagreement internationally around the way in which primary prophylaxis should be incorporated into control strategies. ${ }^{21}$ Everyone seems to be in agreement that promotion of sore throat diagnosis and treatment within existing primary healthcare systems is important. Increasingly, there are calls to accept that microbiological diagnosis of GAS pharyngitis is not likely to be practical. So the ongoing debate to use oral or intramuscular penicillin continues.

\section{B. The role of controlling skin infection}

There is circumstantial evidence from the Aboriginal population in Australia that skin infection may play a role in $\mathrm{RF}$ pathogenesis ${ }^{22}$ which, in turn, raises the possibility that community-based programs to reduce rates of impetigo. ${ }^{23}$ Streptococcal skin infection might, directly or indirectly, have a causative link with $\mathrm{RF}$, or through intervention studies using either controlled trials or observational studies to map the rates of skin infections against rates of $\mathrm{RF} /$ RHD and monitoring changes over time.

\section{Execute what We Know Already into} Practical RHD Control

A. Improved rheumatic heart disease control strategies around the world

It is universally accepted that the most costeffective approach to RHD control is delivery of secondary prophylaxis and improved clinical care of ARF/RHD patients using register-based RHD control programs. [24] Yet, despite the WHO and the WHF recommending these strategies, there has yet to be established a sustained, national program in any developing country. There are examples of successful RHD control programs in some jurisdictions within countries (e.g. Cuba, ${ }^{24}$ and around Chandigarh in northern India ${ }^{25}$ ).

Integrated planning for control programs with primary care and secondary care

RHD control programs currently undertake little in the way of preplanned interactions with primary care staff. So far, nobody has outlined the most effective way that program staff can provide support to primary care staff that improves service delivery.

B. Rheumatic heart disease registers to assess disease outcomes

With a few exceptions, the paucity of populationbased RF/RHD data found in the previous 
attempts at measuring the global disease burden persists today. ${ }^{26}$ More widespread, and better used, RHD register data would allow us to construct sequential cohorts to track mortality, morbidity, and outcomes of valve surgery, analyzed by age group, presence of carditis or chorea at presentation and level of adherence to benzathine penicillin $\mathrm{G}$.

\section{Ways to improve delivery of secondary prophylaxis}

Although there is evidence that establishing register-based control programmes will, in itself, lead to improved adherence with secondary prophylaxis regiments, we are still largely ignorant of the specific ways to dramatically improve the proportion of scheduled benzathine penicillin G injections. There are remarkably few studies of this. Some subthemes here are as follows:

\section{Understanding factors of adherence}

We need qualitative and quantitative research to better understand perceptions and knowledge of patients, families, and health staff about ARF/ RHD and secondary prophylaxis, and practices used by staff and local health systems to deliver care, beyond the small studies available to date. ${ }^{27}$

\section{E. New strategies to improve adherence} Promising strategies from the above studies could then be trialed at the primary care level. There will likely be a range of designs, depending on the setting and level of health center. Some elements that, based on current knowledge, could be considered include (either stand-alone or in combination) implementation of continuous quality improvement approaches, ${ }^{28}$ allocating responsibility for RHD care to particular primary health center staff, streamlining care in clinics so that people are not kept waiting for routine injections, implementing an active recall process and patient empowerment strategies.

\section{F. Effectiveness of comprehensive programs for rheumatic heart disease control}

Successful comprehensive strategies overseas have included registers, support to improve clinical care and delivery of secondary prophylaxis, emphasis on primary prevention (sore throat and skin sore treatment), raising awareness of the symptoms of ARF and the need to seek medical care, improving knowledge of $\mathrm{ARF} / \mathrm{RHD}$, their management, and control among health staff, and broad awareness campaigns in the general community. ${ }^{29}$

\section{The burden and economics of RHD and its control}

To date, there have been only two economic analyses of RHD control, both conducted by the same World Bank group, relying largely on data collected in the USA in the mid- $20^{\text {th }}$ century. ${ }^{30}$ We need comprehensive disease burden estimates (using DALYs) and thorough analyses of the costs of ARF/RHD to model the cost-benefit and cost-effectiveness of different approaches to RF/RHD control.

\section{G. The role of cardiac surgery for RHD in developing countries}

Middle-income countries such as South Africa, India, and Brazil have a growing capacity for cardiac surgery as well as capabilities to assist lower-income countries to operate on RHD patients. The availability of in-country cardiac surgery is increasing in some lower-income countries as well like Bangladesh. In addition, prosthetic valve replacement potentially carries with it significant morbidity and mortality, with devastating complications occurring due to infection, and hemorrhage or thrombosis related to difficulties in managing anticoagulant therapy. ${ }^{31}$

\section{Conclusion:}

Being a third world country Bangladesh is fighting successfully against RF and RHD. Bangladesh can play the role model for those countries who are overburdened with $\mathrm{RF}$ and RHD and their sequels. Being a poor country Bangladesh is using its available resources to curb the incidence and prevalence of $\mathrm{RF}$ and RHD. Only appropriate planning and judicious implementation can reduce huge economic burden for diagnosis and management of such type of disease. Bangladesh needs more population based studies to get the exact information and inference. It is clear that with concerted efforts, strong links to clinical and 
public health infrastructure and with strengthening advocacy and renewed international funding support, we have good prospects of controlling $\mathrm{RF}$ and minimizing the burden of RHD over the next decade.

\section{Competing Interests Statement}

The authors declare no competing interests.

\section{References}

1. Community control of rheumatic heart disease in developing countries: a major public health problem. WHO Chron 1980;34:336-345.

2. Carapetis JR, Steer AC, Mulholland EK, Weber M. The global burden of group A streptococcal diseases. Lancet Infect Dis 2005; 5:685-694.

3. Ahmed J, Zaman MM, Hassan MMM. Prevalence of rheumatic fever and rheumatic heart disease in rural Bangladesh. Trop Doct 2005; 35:160-161.

4. Sadiq M, Islam K, Abid R, Latif F, Rehman AU, Waheed A et al. Prevalence of rheumatic heart disease in school children of urban Lahore. Heart 2009; 95:353-357.

5. Zaman MM, Yoshiike N, Chowdhury AH, Nakayama T, Yokoyama T, Faruque GM et al. Nutritional factors associated with rheumatic fever. J Trop Pediatr 1998; 44:142-147.

6. Carapetis JR, Steer AC, Mulholland EK, Weber M. The global burden of group A streptococcal disease. Lancet Infect Dis 2005; 5:685-694.

7. Tibazarwa KB, Volmink JA, Mayosi BM. Incidence of acute rheumatic fever in the world: a systematic review of population-based studies. Heart 2008; 94:1534-1540.

8. Jackson SJ, Steer AC, Campbell H. Systematic review: Estimation of global burden of non-suppurativesequelae of upper respiratory tract infection: rheumatic fever and post-streptococcal glomerulonephritis. Trop Med Int Health 2011; 16(1):2-11.

9. Khatoon M. Clinical profile of rheumatic fever in some hospitalized children of Bangladesh. Bangladesh Med Res Counc Bull 1985; 11:1-5.

10. Haque KMHSS. Prevention of rheumatic rheumatic heart disease. (Editorial) Bangladesh Heart Journal 1991;6:1-2.

11. Malik A. Congenital and acquired heart diseases (a survey of 7062 persons). Bangladesh Med Res Counc Bull 1976; II: $115-119$.

12. Editorial (anonymous). Decline in rheumatic fever. Lancet $1985 ; 2: 647-647$.

13. Zaman MM, Yoshiike N, Rouf MA, Haque KMHSS, Malik A, Tanaka H. Declining Trend of Rheumatic Fever Observed in a Hospital Specialized in Rheumatic Fever in Bangladesh, 1991-1997. First virtual congress of cardiology.
14. Ahmed J, Zaman MM, Hassan MMM. Prevalence of rheumatic fever and rheumatic heart disease in rural Bangladesh. Trop Doct 2005; 35: 3160-161.

15. Jones TD. Diagnosis of rheumatic fever. JAMA 1944;126:481-4.

16. Heart Foundation of Australia and the Cardiac Society of Australia and New Zealand. Diagnosis and management of acute rheumatic fever and rheumatic heart disease in Australia: An evidence-based review. Melbourne: National Heart Foundation of Australia 2006.

17. Mataika R, Carapetis JR, Kado J, Steer AC. Acute rheumatic fever: An important differential diagnosis of septic arthritis. J Trop Pediatr 2008; 54:205-207.

18. Engel ME, Sr, Vogel J Adeyemo AA, Mayosi BM. Genetic contribution to rheumatic fever: A systematic review and meta-analysis of twin studies.[Presented at the 8th National Congress of the South African Heart Association, Sun City, S Afr Med J 2007; 97:1094.

19. Bryant PA, Robins-Browne R, Carapetis JR, Curtis N. Some of the people, some of the time: Susceptibility to acute rheumatic fever. Circulation 2009; 119:742-753.

20. Paar JA, Berrios NM, Rose JD, Cáceres M, Peña R, Pérez $\mathrm{W}$, et al. Prevalence of rheumatic heart disease in children and young adults in Nicaragua. Am $J$ Cardiol 2010; 105:1809-1814.

21. Karthikeyan G, Mayosi BM. Is primary prevention of rheumatic fever the missing link in the control of rheumatic heart disease in Africa? Circulation 2009; 120:709-713

22. McDonald M, Currie BJ, Carapetis JR. Acute rheumatic fever: A chink in the chain that links the heart to the throat? Lancet Infect Dis 2004; 4:240-245.

23. Lawrence G, Leafasia J, Sheridan J, Hills S, Wate J, Wate $\mathrm{C}$, et al. Control of scabies, skin sores and haematuria in children in the Solomon Islands: Another role for ivermectin. Bull World Health Organ 2005; 83:34-42.

24. WHO Technical Report Series; 923. Geneva: World Health Organization; 2004. WHO Expert Consultation on Rheumatic Fever and Rheumatic Heart Disease (2001: Geneva Switzerland). Rheumatic fever and rheumatic heart disease: Report of a WHO Expert Consultation, Geneva, (29 October - 1 November 2001).

25. Kumar R, Thakur JS, Aggarwal A, Ganguly NK. Compliance of secondary prophylaxis for controlling rheumatic fever and rheumatic heart disease in a rural area of northern India. Indian Heart J 1997; 49:282-288.

26. Tibazarwa KB, Volmink JA, Mayosi BM. Incidence of acute rheumatic fever in the world: A systematic review of population-based studies. Heart 2008; 94:1534-1540. 
27. Mincham CM, Mak DB, Plant AJ. The quality of management of rheumatic fever/heart disease in the Kimberly. Aust N Z J Public Health 2002; 26:417-420.

28. Gardner KL, Dowden M, Togni S, Bailie R. Understanding uptake of continuous quality improvement in Indigenous primary health care: Lessons from a multi-site case study of the Audit and Best Practice for Chronic Disease project. Implement Sci 2010; 5:21.

29. Bach JF, Chalons S, Forier E, Elana G, Jouanelle J, Kayemba $\mathrm{S}$, et al. 10-year educational programme aimed at rheumatic fever in two French Caribbean islands. Lancet 1996; 347:644.

30. Michaud C, Rammohan R, Narula J. Cost-effectiveness analysis of intervention strategies for reduction of the burden of rheumatic heart disease. In: Narula J, Virmani R, Reddy KS, Tandon R, editors. Rheumatic fever. Washington DC: American Registry of Pathology 1999;. 485-497.

31. Carapetis JR, Powers JR, Currie BJ. Outcomes of cardiac valve replacement for rheumatic heart disease in Aboriginal Australians. Asia Pac Heart J 2000; 8:138-147. 